\title{
Distinguishable Immunologic Characteristics of COVID-19 Patients with Comorbid Type 2 Diabetes Compared with Nondiabetic Individuals
}

\author{
Ruxing Zhao, ${ }^{1,2,3}$ Yujing Sun, ${ }^{1,2,3}$ Yongyuan Zhang, ${ }^{4}$ Weili Wang, ${ }^{1}$ Shouyu Wang, \\ Chuang Wang, ${ }^{1}$ Jinbo Liu, ${ }^{1}$ Ling Gao, ${ }^{5}$ Zhao Hu, ${ }^{6}$ Jianchun Fei, ${ }^{7}$ Xinguo Hou ${ }^{D},{ }^{1,2,3}$ \\ Huizhen Zheng $\mathbb{D}{ }^{1}$ and Li Chen $\mathbb{D}^{1,2,3}$ \\ ${ }^{1}$ Department of Endocrinology, Qilu Hospital, Cheeloo College of Medicine, Shandong University, Jinan 250012, China \\ ${ }^{2}$ Institute of Endocrine and Metabolic Diseases of Shandong University, Jinan 250012, China \\ ${ }^{3}$ Jinan Clinical Research Center for Endocrine and Metabolic Diseases, Jinan 250012, China \\ ${ }^{4}$ Division of Medical Affairs, Qilu Hospital of Shandong University, Jinan 250012, China \\ ${ }^{5}$ Department of Endocrinology, Renmin Hospital of Wuhan University, Wuhan 430060, China \\ ${ }^{6}$ Department of Nephrology, Qilu Hospital, Cheeloo College of Medicine, Shandong University, Jinan 250012, China \\ ${ }^{7}$ Department of Anesthesiology, Qilu Hospital, Cheeloo College of Medicine, Shandong University, Jinan 250012, China
}

Correspondence should be addressed to Xinguo Hou; houxinguo@sdu.edu.cn, Huizhen Zheng; zhenghuizhen1@163.com, and Li Chen; chenli3@medmail.com.cn

Received 11 June 2020; Revised 9 August 2020; Accepted 7 September 2020; Published 29 September 2020

Academic Editor: Paola Migliorini

Copyright (C) 2020 Ruxing Zhao et al. This is an open access article distributed under the Creative Commons Attribution License, which permits unrestricted use, distribution, and reproduction in any medium, provided the original work is properly cited.

\begin{abstract}
Background. COVID-19 caused by severe acute respiratory syndrome coronavirus 2 (SARS-CoV-2) has threatened every civilian as a global pandemic. The immune system poses the critical interactive chain between the human body and the virus. Here, we make efforts to examine whether comorbidity with type 2 diabetes (T2D) affects the immunological response in COVID-19 patients. Methods. We conducted a retrospective pilot study investigating immunological characteristics of confirmed cases of COVID-19 with or without comorbid T2D. Two subcohorts of sex- and age-matched participants were eligible for data analysis, of which 33 participants were with T2D and the remaining 37 were nondiabetic (NDM). Cellular immunity was assessed by flow cytometric determination of surface markers including CD3, CD4, CD8, CD19, CD16, and CD56 in peripheral blood. Levels of $\mathrm{C}$ reactive protein, immunoglobulin (IgG, IgM, IgA, and $\operatorname{IgE}$ ), and complements $(\mathrm{C} 3, \mathrm{C} 4)$ were detected by rate nephelometry immunoassay. And Th1/Th2 cytokines (IL-2, IL-4, IL-6, IL-10, TNF- $\alpha$, and IFN- $\gamma$ ) were detected by Cytometric Bead Array. Results. Neutrophil counts were found to be significantly higher in the T2D group than in the NDM group and had a significant relevance with clinical severity. Lymphocyte frequencies showed no significant differences in the two groups. However, the proportions and absolute counts of T, Tc, Th, and NK cells decreased in both groups to different degrees. An abnormal increase in neutrophil count and a decrease in lymphocyte subpopulations may represent risk factors of COVID-19 severity. The level of IgG, IgM, IgA, C3, and C4 showed no significant difference between the two groups, while the IgE levels were higher in the T2D group than in the NDM group $(p<0.05)$. Th1 cytokines including IFN- $\gamma$, TNF- $\alpha$, and IL-6, as well as CRP, appeared significantly higher in the T2D group. Conclusions. The COVID-19 patients comorbid with T2D demonstrated distinguishable immunological parameters, which represented clinical relevancies with the predisposed disease severity in T2D.
\end{abstract}

\section{Introduction}

From January, 2020, we have been facing an unprecedented outbreak of coronavirus infectious disease-19 (COVID-19), which is now threatening every civilian in the world [1]. COVID-19 is caused by a novel severe acute respiratory syndrome coronavirus 2 (SARS-CoV-2) $[2,3]$. Although COVID-19 leads to mild flu-like symptoms in the majority 
of affected patients, the disease may cause severe or even frequently lethal complications such as acute respiratory distress syndrome (ARDS) and multiorgan dysfunction (MODS) $[1,2,4,5]$. And the coronavirus, including SARSCoV-2, may likely pose a continuous threat to human health in the future [6]. Considering the infectivity of the virus, we must be prepared for further challenges. More knowledge on the pathogen and the host immune response is needed to develop more effective public health measures. Multimorbidity is also among the most important consideration for future control of the pandemic.

In parallel, T2D is one of the largest noncommunicable disease epidemics worldwide, whose increase is also in an uncontrolled and explosive manner $[7,8]$. T2D is well established with alterations in both adaptive and innate immune systems, thus increasing the risk of susceptibility to most kinds of infections $[9,10]$. Up to now, T2D is one of the most important comorbidities linked to the severity of all three known human pathogenic coronavirus infections, including SARS-CoV-2 [2, 10-12]. Previous data suggested that diabetes might likely be one of the most important comorbidities linked with COVID-19 [13]. It is known that $20-50 \%$ of patients in the current coronavirus COVID-19 pandemic had diabetes $[14,15]$. Patients with T2D are supposed to have an increased risk of more severe outcome during infection [15]. Thus, an understanding of the intricate pathways responsible for the pathogenesis and complications in T2D and the development of strategies to enhance and stabilize the immune system are both urgently needed to prevent coinfections and comorbidities in COVID-19 patients with T2D $[11,15]$. There are now more and more studies comprehensively demonstrating the clinical and laboratory parameters in COVID-19 with and without diabetes [16-19]. However, data on specific immunological changes in COVID-19 patients comorbid with T2D are yet limited [12].

In this retrospective pilot study, we examined the population of leukocytes and lymphocyte subsets, humoral immunity, infection-related biomarkers, and inflammatory cytokines in two subcohorts of sex- and age-matched clinically and laboratory-confirmed cases of COVID-19. We made efforts to check whether comorbidity with T2D affects the immunological parameters during diagnosis and management of COVID-19.

\section{Materials and Methods}

2.1. Participants and Ethic Statement. This study was a retrospective cohort study of hospitalized patients admitted to the People's Hospital of Wuhan University, one of the major hospitals nationally designated to provide medical care for COVID-19 patients in Wuhan, from February 5, 2020, to March 10, 2020. All participants enrolled were confirmed cases of COVID-19 diagnosed in compliance with the Guidelines for Diagnosis and Management of COVID-19 (6th edition) issued by the National Health Committee of China. Respiratory specimens were collected and then shipped to designated authoritative laboratories to detect the SARSCoV-2 as previously reported $[1,5]$. The positive finding of viral nucleic acid was considered essential for the enrollment.
A detailed clinical record was registered for each subject, including history of COVID-19 disease, positive physical examination findings, and laboratory examinations. And a total of 70 participants were eligible and consecutively enrolled for the study, including two subcohorts of sex- and age-matched patients with and without T2D (referred as the T2D and NDM groups, $n=37$ and $n=33$, respectively) in search of immunological differences.

Patients with uncertain diagnosis of either type 2 diabetes (confirmed or suspected type 1 diabetes or other special types of diabetes) or COVID-19 or other unknown situations were excluded from the study. Exclusion criteria also included any clues of autoimmune disorders or tumors. All participants did not receive immunosuppressive or immunomodulatory drugs for at least 3 months before blood sampling.

This experiment was approved by the ethics committee of Qilu Hospital of Shandong University (No. 2020010). The data are kept anonymous; therefore, the requirement of written informed consent was waived. The study was conducted in compliance with the Declaration of Helsinki principles for ethical research.

2.2. Clinical Variables. Detailed clinical data were collected, including age, sex, exposure history, comorbid conditions, symptoms, and laboratory results. Clinical data including classification of disease severity and outcome were obtained from electronic medical records. Disease severity was categorized into mild, moderate, severe, and critically ill cases according to the above Guidelines for Diagnosis and Management of COVID-19. Due to the low incidence rate of critically ill cases in the cohort, we combined severe and critically ill cases as one classification-severe. Specimens, including sputum, blood, and urine were cultured to identify pathogenic bacteria or fungi secondary to or mixed with the SARS-CoV-2 infection. Mixed bacterial infection was diagnosed depending on both clinical manifestations and positive laboratory findings of microbiological examination and/or significantly increased procalcitonin (PCT $>0.1 \mathrm{ng} / \mathrm{mL}$ ). Well-trained attending physicians were responsible for the diagnostic procedures, interpretation of laboratory analyses, and clinical decision during the patients' in-hospital stay. The data collection forms were reviewed independently by two researchers and analyzed in a blinded manner.

2.3. Laboratory Evaluation. Cellular immunity was assessed by multicolor flow cytometric determination of surface markers using human monoclonal anti-CD3-fluorescein isothiocyanate (FITC), anti-CD4-phycoerythrin (PE), antiCD8-allophycocyanin (APC), anti-CD19-PE, anti-CD16APC, and anti-CD56-PE antibodies (BD Multitest), for determination of proportions and numbers of total $\mathrm{T}$ (CD3+), helper $\mathrm{T}$ (Th, $\mathrm{CD} 3+\mathrm{CD} 4+)$, cytotoxic $\mathrm{T}(\mathrm{Tc}$, CD3+CD8+), Natural Killer (NK, CD3-CD16+CD56+), and $\mathrm{B}$ (CD3-CD19+) cell subsets. All samples were examined by a BD FACSCanto II Flow Cytometer. Data were analyzed by FlowJo v10.0.

Serum levels of $\mathrm{C}$ reactive protein (hypersensitive, hsCRP), immunoglobulin (IgG, $\operatorname{IgM}, \operatorname{IgA}$, and $\operatorname{IgE}$ ), and complements $(\mathrm{C} 3, \mathrm{C} 4)$ were detected by rate nephelometry 
immunoassay ( $\mathrm{N}$ Antiserum to Human Ig Kit series, Siemens, Germany). The plasma levels of cytokines (IL-2, IL-4, IL-6, IL-10, TNF- $\alpha$, and IFN- $\gamma$ ) were detected by Cytometric Bead Array using the human Th1/2 cytokine kit II (BD Ltd., USA). All tests were conducted according to the manufacturer's instructions.

2.4. Statistical Analysis. Briefly, continuous parameters were presented as the mean \pm SD or median according to data distribution. The classification variable was presented as a count (\%). The statistical difference between two groups was determined by nonpaired Student's $t$-test unless the data were not normally distributed, in which case Mann-Whitney's $U$ test was used instead. The chi-squared goodness-of-fit (Fisher's exact) test was used for the comparison of incident rates and proportions for categorical variables. The odds ratio was calculated in a $2 \times 2$ arranged table with Fisher's exact test. Spearman's correlation analysis was conducted between pairs of parameters to evaluate preliminarily the possible correlation. Ordinal multinomial logistic regression analysis was used to determine the associations of clinical and laboratory parameters with disease severity (mild, moderate, and severe/critical ill). For binary regression analysis, mild and moderate cases were defined as nonsevere and severe and critically ill cases were defined as severe. SPSS18.0 or GraphPad Prism 5.0 was used to perform all tests and generate values. A $p$ value of less than 0.05 was considered statistically significant.

\section{Results}

3.1. Baseline Demographic Characteristics of COVID-19 Patients with and without T2D. Seventy patients met the inclusion criteria and were eligible for the study, of which 37 patients $(52.9 \%)$ with diabetes belonged to the T2D group and 33 patients $(47.1 \%)$ without diabetes belonged to the NDM group, respectively. There were no significant differences between the two groups among the demographic parameters, including age (average age or age distribution) and sex. Participants in both groups had underlying chronic medical conditions including a high prevalence of cardiovascular diseases $(42.4 \%$ and $77.8 \%$, respectively, including hypertension in this category). And we did find statistically significant differences concerning the comorbidity rates of these chronic diseases in our study cohort (shown in Table 1).

Previous literature had suggested that T2D might be associated with a higher risk of developing more severe types of infection $[2,11,15]$. However, there were no systemic data supporting the fact. In this retrospective cohort study, we also observed a significantly lesser proportion of mild types (18.9\% vs. $48.5 \%)$ and more cases of severe types $(24.3 \%$ vs. $12.1 \%$ ) of infection in the T2D group compared with the NDM group $(p=0.038)$. In ordinal multinomial logistic regression analysis, comorbidity with $\mathrm{T} 2 \mathrm{D}$ was for the first time proven to be a significant risk factor for having more severe types of infection (OR: 3.498 (1.369-8.938); $p=$ $\left.0.009^{*}\right)$. And the association was still statistically significant after adjusting for age and sex (OR: 3.388 (1.320-8.698); $\left.p=0.011^{*}\right)$. Besides, patients from the T2D group also had a significantly higher risk of mixed bacterial infection than patients from the NDM group (see Table 1).

3.2. Distinguished Hematological Characteristics of COVID19 in the T2D Group and Its Clinical Relevance. Here, we compare the hematological parameters between the two subcohorts. Patients with COVID-19 often had anemia, leukocytopenia, lymphocytopenia, and an increased monocyte count as previously reported $[1,2,20]$. Surprisingly, there were significant elevations in blood levels of leukocyte and neutrophil counts compared with NDM (shown in Table 2). Eosinophil count was decreased in T2D compared with NDM (0.08 vs. $\left.0.02 ; p=0.038^{*}\right)$. The T2D group also showed a significantly higher incidence of abnormal leukocytosis ( $16.22 \%$ vs. $0 \%$; $\left.p=0.0294^{*}\right)$ and an even more remarkably higher incidence of neutrophilia (OR: 11.85 (1.705 to 132.3 ); $p=0.0074^{*}$ ). However, blood levels of lymphocyte count seemed lower in the T2D group than in the NDM group, but the current data failed to provide statistical significance ( 0.98 vs. 1.43 ; $p=0.097)$. There were no differences in monocyte ( 0.45 vs. $0.52 ; p=0.2641)$ or basophil count ( 0.02 vs. $0.03 ; p=0.960)$ between NDM and T2D patients. Since our data indicated that COVID-19 patients comorbid with T2D had a significantly higher incidence of mixed bacterial infection, we further analyzed whether the increased incidence of neutrophilia was associated with bacterial infection in a $2 \times 2$ arranged table with Fisher's exact test. The current data did not show correlation between neutrophilia and bacterial infection in either total participants or two subcohorts $(p=0.7601)$. The abnormal increase in neutrophil count might thus be associated with comorbidity with T2D and independent of bacterial infection.

The results above indicate distinguishable changes in the white cell subpopulation of COVID-19 patients with T2D. Correlation analysis also revealed possible links between an abnormal increase in neutrophil count with decreased $\mathrm{T}$ $\left(r=-0.348 ; p=0.003^{*}\right)$, Th $\left(r=-0.342 ; p=0.004^{*}\right)$, Tc $\left(r=-0.344 ; p=0.004^{*}\right)$, and $\mathrm{B}\left(r=-0.288 ; p=0.016^{*}\right)$ cell count and $\mathrm{NK}\left(r=-0.252 ; p=0.035^{*}\right)$ cell proportion, as well as a positive link with $\operatorname{IgG}\left(r=0.249 ; p=0.043^{*}\right), \operatorname{IgE}$ $\left(r=0.316 ; p=0.024^{*}\right)$, and IFN- $\gamma \quad\left(r=0.305 ; p=0.010^{*}\right)$ (shown in Figure 1). As increases in neutrophil counts and neutrophil-to-lymphocyte ratio (NLR) were most recently established as predicative markers of severity during SARSCoV-2 infection [20], we further conducted logistic regression analysis to verify whether neutrophilia represents an independent risk factor for adverse outcomes of COVID-19 in T2D. Neutrophil count was established as an independent risk factor associated with severe (and critically ill) types of COVID-19 in a binary univariate regression model (OR: $\left.1.23(1.029-1.527) ; p=0.0314^{*}\right)$ after adjusting for age, sex, and mixed bacterial infection, which indicated that an increase of one thousand neutrophils per microliter of peripheral blood was associated with a 1.23-fold increase of risk of developing severe types (instead of mild or moderate types) of COVID-19. An increase in NLR did not represent a higher risk or significance in our study (OR: 1.095 (0.986$1.235) ; p=0.1027)$. Interestingly, when comorbidity with T2D was introduced into the model, neutrophil count would 
TABLE 1: Basal characteristics of patients infected with COVID-19.

\begin{tabular}{|c|c|c|c|}
\hline & $\operatorname{NDM}(n=33)$ & $\mathrm{T} 2 \mathrm{D}(n=37)$ & $p$ value \\
\hline \multicolumn{4}{|l|}{ Age distribution } \\
\hline $25-49, n(\%)$ & $6(19.6 \%)$ & $5(13.5 \%)$ & 0.592 \\
\hline $50-64, n(\%)$ & $10(30.3 \%)$ & $15(40.5 \%)$ & 0.372 \\
\hline$\geq 65, n(\%)$ & $17(51.5 \%)$ & $17(45.9 \%)$ & 0.641 \\
\hline Age (years) & $62.1 \pm 16.2$ & $63.4 \pm 12.8$ & 0.690 \\
\hline \multicolumn{4}{|l|}{ Sex } \\
\hline Female, $n(\%)$ & $15(45.5 \%)$ & $18(48.6 \%)$ & 0.699 \\
\hline Severity classification & & & $0.038^{*}$ \\
\hline Mild type, $n(\%)$ & $16(48.5 \%)$ & $7(18.9 \%)$ & - \\
\hline Moderate type, $n(\%)$ & $13(39.4 \%)$ & $20(54.1)$ & - \\
\hline Severe cases, $n(\%)$ & $4(12.1 \%)$ & $9(24.3 \%)$ & - \\
\hline Critically ill type, $n(\%)$ & $0(0.0 \%)$ & $1(2.7 \%)$ & - \\
\hline \multicolumn{4}{|l|}{ Comorbidities at admission } \\
\hline Mixed bacterial infection, $n(\%)$ & $4(12.1 \%)$ & $12(32.3 \%)$ & $0.043^{*}$ \\
\hline Cardiovascular diseases, $n(\%)$ & $14(42.4 \%)$ & $21(77.8 \%)$ & 0.231 \\
\hline Digestive system disease, $n(\%)$ & $2(6.1 \%)$ & $3(8.1 \%)$ & 0.895 \\
\hline Respiratory system diseases, $n(\%)$ & $2(6.1 \%)$ & $3(8.1 \%)$ & 0.895 \\
\hline Chronic kidney disease, $n(\%)$ & $2(6.1 \%)$ & $5(18.5 \%)$ & 0.521 \\
\hline Chronic liver disease, $n(\%)$ & $1(3.0 \%)$ & $1(2.7 \%)$ & 0.628 \\
\hline HIV infection, $n(\%)$ & $0(0.0 \%)$ & $0(0.0 \%)$ & 1.000 \\
\hline Septic shock, $n(\%)$ & $0(0.0 \%)$ & $0(0.0 \%)$ & 1.000 \\
\hline
\end{tabular}

TABLE 2: Laboratory findings of white cell subpopulations in patients with diabetes or not infected with COVID-19.

\begin{tabular}{|c|c|c|c|c|c|c|c|c|}
\hline \multirow{2}{*}{$\begin{array}{l}\text { Variables } \\
\left(\times 10^{9} / \mathrm{L}\right)\end{array}$} & \multirow{2}{*}{ Normal range } & \multicolumn{3}{|c|}{$\operatorname{NDM}(n=33)$} & \multicolumn{3}{|c|}{$\mathrm{T} 2 \mathrm{D}(n=37)$} & \multirow{2}{*}{$p$ value } \\
\hline & & Median & Increased no. (\%) & Decreased no. (\%) & Median & Increased no. (\%) & Decreased no. (\%) & \\
\hline WBC & $3.5-9.5$ & 4.74 & $0(0.0 \%)$ & $5(15.2 \%)$ & 6.05 & $6(16.2 \%)$ & $2(5.4 \%)$ & $0.015^{*}$ \\
\hline $\mathrm{NEU}$ & $1.8-6.3$ & 2.50 & $1(3.0 \%)$ & $2(6.1 \%)$ & 4. 10 & $10(27.0 \%)$ & $0(0.0 \%)$ & $<0.001^{*}$ \\
\hline LYM & $1.1-3.2$ & 1.43 & $0(0.0 \%)$ & $17(39.5 \%)$ & 0.98 & $3(8.1 \%)$ & $18(48.7 \%)$ & 0.097 \\
\hline ESO & $0.02-0.52$ & 0.08 & $0(0.0 \%)$ & $8(24.3 \%)$ & 0.02 & $0(0.0 \%)$ & $16(43.2 \%)$ & $0.038^{*}$ \\
\hline BAS & $0-0.06$ & 0.02 & $2(6.1 \%)$ & $0(0.0 \%)$ & 0.03 & $1(2.7 \%)$ & $0(0.0 \%)$ & 0.960 \\
\hline $\mathrm{MON}$ & $0.1-0.6$ & 0.45 & $7(21.2 \%)$ & $0(0.0 \%)$ & 0.52 & $11(29.7 \%)$ & $0(0.0 \%)$ & 0.264 \\
\hline
\end{tabular}

${ }^{1}$ WBC: white blood cell count; NEU: neutrophil count; LYM: lymphocyte count; ESO: eosinophil count; BAS: basophil count; MON: monocyte count. ${ }^{2}$ Note that the $p$ values were calculated by comparisons of absolute values (medians or means) in this table.

lose its significant relevance. These preliminary data suggested that an abnormal increase in neutrophil count and its contribution to COVID-19 severity may be associated with dysregulated immune response in T2D.

3.3. Differences in Cellular Immunity in the T2D Group Compared with the NDM Group and Its Clinical Relevance. As shown above, the total lymphocyte levels had no significant difference between the two groups. However, both groups had a high incidence of lymphopenia. Previous data had revealed that dysregulation of immune response, especially $\mathrm{T}$ lymphocytes, might be highly involved in the pathological process of COVID-19 [20]. We felt obliged to further compare and analyze the subsets of lymphocytes in two subcohorts. Sustained decrease in total T (CD3+) cells, Th
(CD4+) cells, Tc (CD8+) cells, and NK (CD16+CD56+) cells was often observed in the current cohort of COVID-19 patients. The decrease of absolute amounts (cells per microliter of peripheral blood) of total T cells, Tc cells, Th cells, and NK cells was more significantly remarkable in the T2D group than in the NDM group (shown in Table 3 and Figure 2(a)). A decrease in Tc cells appeared to be more dominant; thus, the $\mathrm{Th} / \mathrm{Tc}(\mathrm{CD} 4+/ \mathrm{CD} 8+)$ ratio was often increased in COVID19 patients. However, we did not observe a significant difference in the Th/Tc ratio between the two groups. Some of the patients had an increased number and/or proportion of $\mathrm{B}$ cells, which might represent a normal response to viral infection. Here, we found statistical difference in the proportion but not in the number of B cells between the T2D group and the NDM group (see Table 3 and Figure 2). 


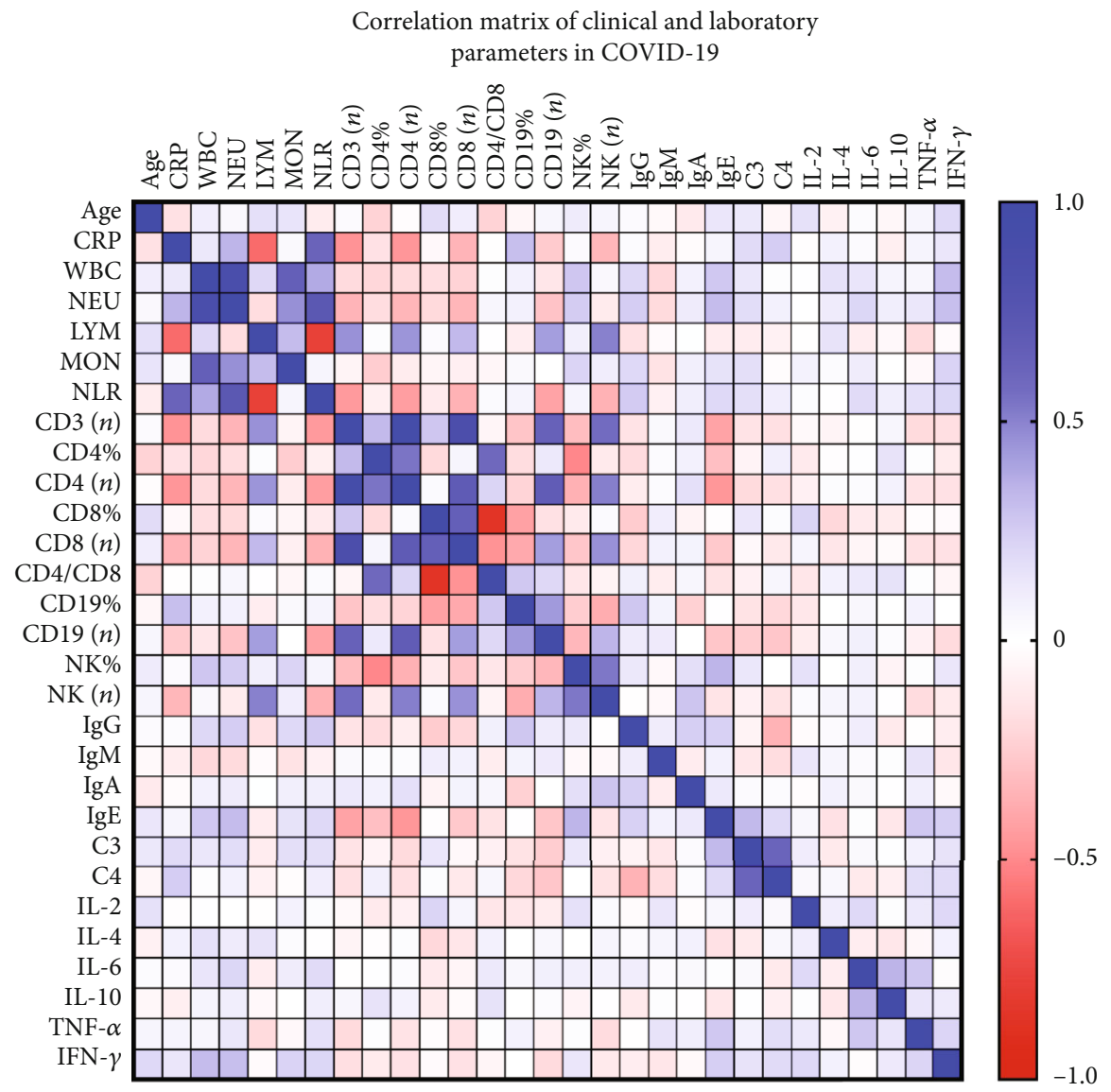

Figure 1: Correlation matrix of clinical and laboratory parameters in COVID-19 Spearman's correlation coefficients between two pairs of variables is shown in the heat map. The correlation coefficients are represented in terms of the change of the intensity of red/blue color, as shown in the color bar.

TABLe 3: Status of cellular immunity in patients with and without type 2 diabetes.

\begin{tabular}{|c|c|c|c|c|c|c|c|c|}
\hline \multirow{2}{*}{ Variables } & \multirow{2}{*}{ Normal range } & \multicolumn{3}{|c|}{$\operatorname{NDM}(n=33)$} & \multicolumn{3}{|c|}{$\mathrm{T} 2 \mathrm{D}(n=37)$} & \multirow{2}{*}{$p$ value } \\
\hline & & Median & Increased no. (\%) & Decreased no. (\%) & Median & Increased no. (\%) & Decreased no. (\%) & \\
\hline $\mathrm{T}(\%)$ & $56-86$ & 67.0 & $0(0.0 \%)$ & $4(12.1 \%)$ & 62.9 & $0(0.0 \%)$ & $9(23.1 \%)$ & 0.059 \\
\hline $\mathrm{T}(n / \mu \mathrm{L})$ & $723-2737$ & 983 & $0(0.0 \%)$ & $9(27.3 \%)$ & 679 & $0(0.0 \%)$ & $19(48.7 \%)$ & $0.004^{*}$ \\
\hline $\operatorname{Th}(\%)$ & $33-58$ & 41.1 & $1(3.0 \%)$ & $6(18.2 \%)$ & 42.0 & $0(0.0 \%)$ & $1(2.6 \%)$ & 0.926 \\
\hline $\operatorname{Th}(n / \mu \mathrm{L})$ & $404-1612$ & 604 & $0(0.0 \%)$ & $8(24.2 \%)$ & 451 & $0(0.0 \%)$ & $6(15.4 \%)$ & $0.029^{*}$ \\
\hline $\mathrm{Tc}(\%)$ & $13-39$ & 24.0 & $2(6.1 \%)$ & $4(12.1 \%)$ & 18.7 & $0(0.0 \%)$ & $18(46.2 \%)$ & $0.017^{*}$ \\
\hline $\mathrm{Tc}(n / \mu \mathrm{L})$ & $220-1129$ & 328 & $0(0.0 \%)$ & $12(36.4 \%)$ & 203 & $0(0.0 \%)$ & $22(66.7 \%)$ & $<0.001^{*}$ \\
\hline $\mathrm{Th} / \mathrm{Tc}$ & $0.9-2.0$ & 2.12 & $15(45.5 \%)$ & $3(9.1 \%)$ & 2.54 & $22(56.4 \%)$ & $0(0.0 \%)$ & 0.221 \\
\hline B (\%) & $5-22$ & 13.4 & $4(12.1 \%)$ & $0(0.0 \%)$ & 19.4 & $12(30.8 \%)$ & $0(0.0 \%)$ & $0.018^{*}$ \\
\hline $\mathrm{B}(n / \mu \mathrm{L})$ & $80-616$ & 187 & $1(3.0 \%)$ & $2(6.1 \%)$ & 170.3 & $1(2.6 \%)$ & $5(15.2 \%)$ & 0.384 \\
\hline NK (\%) & $5-26$ & 17.3 & $2(6.1 \%)$ & $0(0.0 \%)$ & 16.4 & $3(7.7 \%)$ & $3(7.7 \%)$ & 0.810 \\
\hline $\mathrm{NK}(n / \mu \mathrm{L})$ & $84-724$ & 242 & $0(0.00 \%)$ & $2(6.1 \%)$ & 186 & $1(2.6 \%)$ & $14(35.9 \%)$ & $0.045^{*}$ \\
\hline
\end{tabular}

Similar tendencies were also observed in the incidences of parameter abnormity between the two groups. The degree and incidence of decreased Tc cells turned out to be a more distinguished dysregulated cellular component in COVID19, which is consistent with previous studies [5, 21]. 36.4\% of patients without diabetes and above half $(66.7 \%)$ of T2D patients had a decreased Tc cell count $\left(p=0.040^{*}\right)$. Patients from the T2D group also had a significantly higher incidence of decreased Tc cell proportion than those from the NDM group $\left(46.2 \%\right.$ vs. $\left.12.1 \% ; p=0.008^{*}\right)$. The T2D group also had a remarkably higher incidence of decreased NK cell count than the NDM group (35.89\% vs. $\left.6.06 \% ; p=0.001^{*}\right)$. 


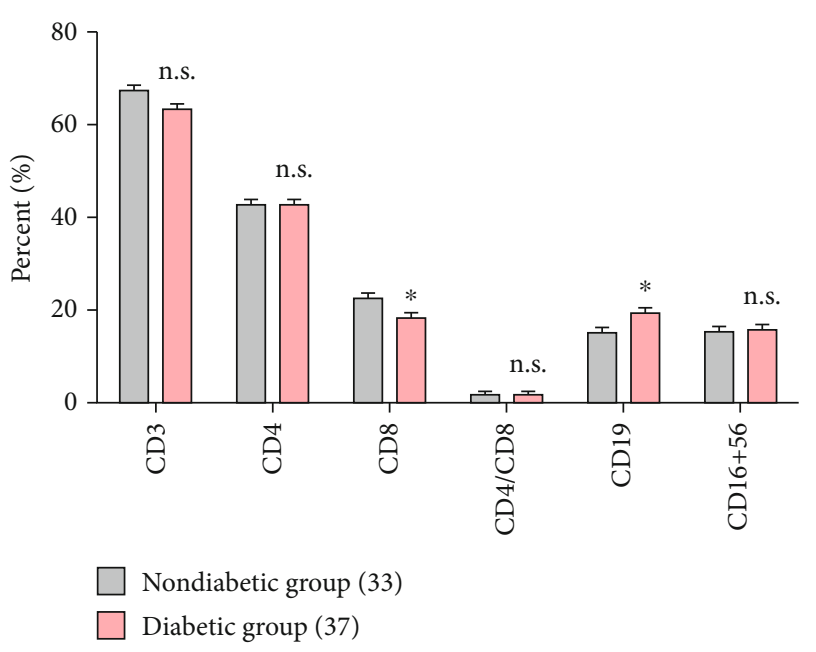

(a)

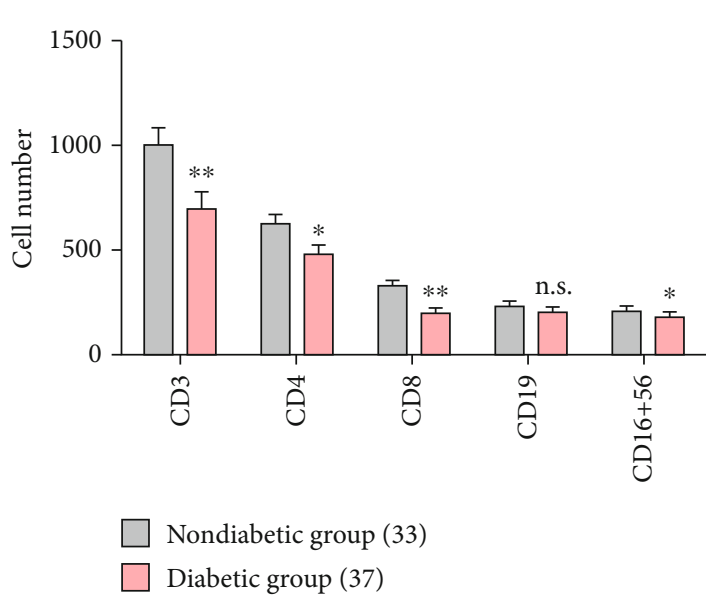

(b)

FIgURe 2: Comparisons of proportions and cell counts of lymphocyte subpopulations between COVID-19 patients with and without diabetes. Note: n.s.: $p>0.05 ;{ }^{*} p<0.05 ;{ }^{* *} p<0.01$.

However, when considering the frequencies of the cell subpopulation, there were less patients with decreased Th cell proportion in the T2D group than in the NDM group $\left(2.56 \%\right.$ vs. $\left.18.2 \% ; p=0.044^{*}\right)$. Although the T2D group had a lower median level of Th cell count, they had a lower incidence of decreased Th cell count at the same time (see Table 3). Currently, using ordinal multinomial logistic regression models, univariate analysis revealed that only $\mathrm{Th}$ cell count might have potential negative relevance (OR: $\left.0.176(0.034-0.907) ; p=0.0379^{*}\right)$ with the COVID-19 severity. When adjusted by age and sex, the relevance remained statistically significant and even more remarkable (OR: $\left.0.137(0.024-0.790) ; p=0.0261^{*}\right)$. Age and sex might have an influence on the absolute number of Th cell count. When comorbidity with T2D was introduced into the model, T2D still represented a significant risk factor for more severe types of disease (OR: $2.760(1.049-7.266)$; $\left.p=0.0398^{*}\right)$, but again Th cell count lost its significance $(p=0.086)$. In the correlation analysis, Th cell count correlated with many other parameters involving antiviral response as well as inflammatory markers. It positively correlated with lymphocyte count, Tc cell count, B cell count, and NK cell count significantly, but it negatively correlated with CRP levels and neutrophil count (shown in Figure 1). As an important regulatory immune component, Th cells may play a double-edged role in antiviral response and uncontrolled inflammation. The abovementioned data supported the possibility that there were discrepant and even divergent cellular immune responses to SARS-CoV-2 infection in patients comorbid with T2D.

\subsection{Differences in Serum Immunoglobulin and Complements} in the T2D Group Compared with the NDM Group. Humoral immunity plays important roles in antiviral response. In this study, we observed changes in serum levels of immunoglobulin and complements in COVID-19. Patients had more often increased levels of IgG, IgE, and complement C4, but decreased levels of C3 (shown in Table 4). The average levels of the IgG, IgM, IgA, C3, and C4 showed no significant difference between the two groups. But abnormally higher $\operatorname{IgE}$ levels were found to be statistically significant in the T2D group compared with the NDM group. And there were also more patients with an increased level of IgG in the T2D group than in the NDM group. Moreover, serum IgE negatively correlated with Th and B cell counts significantly but positively correlated with neutrophil count $\left(p<0.05^{*}\right)$. In search of the clinical relevance of the immunoglobulin and complements with disease severity, we further conducted univariate logistic regression analysis involving $\operatorname{IgG}, \operatorname{IgM}$, $\operatorname{IgA}, \operatorname{IgE}, \mathrm{C} 3$, and $\mathrm{C} 4$, but none of them indicated statistical significance (data not shown).

3.5. Differences in Serum Th1/Th2 Cytokines of Patients in the T2D Group Compared with the NDM Group. As cytokine storms played a critical role in the deterioration of COVID19, we also detected the Th1/Th2 cytokines including IL-2, IL-4, IL-6, IL-10, TNF- $\alpha$, and IFN- $\gamma$ in patients infected with COVID-19. As shown in Table 5, we did not find statistical difference in the levels of the Th2 cytokines including IL-2, IL-4, and IL-10. However, serum levels of IFN- $\gamma$, TNF- $\alpha$, IL-6, and CRP were all significantly higher in the T2D group than in the NDM group $(p=0.005, p=0.0001, p=0.008$, and $p=0.015$, respectively). What is more, increased serum levels of CRP correlated significantly not only with increased neutrophil count (and NLR) but also with decreased lymphocyte, $\mathrm{T}$ cell, Th, Tc, B cell, and NK cell counts $\left(p<0.05^{*}\right.$, shown in Figure 1). However, none of them showed statistical significance considering the clinical relevance of the cytokines with COVID-19 severity by univariate logistic regression analysis.

\section{Discussion}

The ongoing pandemic of COVID-19 is now a global healththreatening crisis $[2,3]$. Within the past half year, we have accumulated limited knowledge of the novel infectious disease. The immune response is believed to be most involved 
TABLE 4: Serum levels of immunoglobulins and complements in patients with and without type 2 diabetes.

\begin{tabular}{|c|c|c|c|c|c|c|c|c|}
\hline \multirow{2}{*}{ Variables } & \multirow{2}{*}{ Normal range } & \multicolumn{3}{|c|}{$\operatorname{NDM}(n=33)$} & \multicolumn{3}{|c|}{$\mathrm{T} 2 \mathrm{D}(n=37)$} & \multirow{2}{*}{$p$ value } \\
\hline & & Median & Increased no. (\%) & Decreased no. (\%) & Median & Increased no. (\%) & Decreased no. (\%) & \\
\hline $\operatorname{IgG}(\mathrm{g} / \mathrm{L})$ & $7-16$ & 23.83 & $2(6.5 \%)$ & $1(3.2 \%)$ & 25.2 & $10(27.8 \%)$ & $1(2.8 \%)$ & 0.547 \\
\hline $\operatorname{IgM}(g / L)$ & $0.4-2.3$ & 1.03 & $0(0.0 \%)$ & $1(3.2 \%)$ & 1.02 & $2(5.6 \%)$ & $0(0.0 \%)$ & 0.680 \\
\hline $\operatorname{IgA}(\mathrm{g} / \mathrm{L})$ & $0.7-4.0$ & 2.87 & $2(6.5 \%)$ & $0(0.0 \%)$ & 3.13 & $8(22.2 \%)$ & $1(2.8 \%)$ & 0.457 \\
\hline $\operatorname{IgE}(\mathrm{IU} / \mathrm{mL})$ & $<100$ & 88.5 & $5(16.1 \%)$ & $0(0.0 \%)$ & 163.4 & $13(36.1 \%)$ & $0(0.0 \%)$ & $0.002^{*}$ \\
\hline C3 (g/L) & $0.9-1.8$ & 1.03 & $0(0.0 \%)$ & $3(9.7 \%)$ & 1.01 & $1(2.8 \%)$ & $7(19.4 \%)$ & 0.402 \\
\hline $\mathrm{C} 4(\mathrm{~g} / \mathrm{L})$ & $0.1-0.4$ & 0.23 & $4(12.9 \%)$ & $1(3.2 \%)$ & 0.25 & $3(8.3 \%)$ & $1(2.8 \%)$ & 0.839 \\
\hline
\end{tabular}

TABLE 5: Serum levels of Th1/Th2 cytokines in patients with and without type 2 diabetes.

\begin{tabular}{|c|c|c|c|c|c|c|}
\hline \multirow{2}{*}{ Variables $(\mathrm{pg} / \mathrm{mL})$} & \multirow{2}{*}{ Normal range } & \multicolumn{2}{|c|}{$\operatorname{NDM}(n=33)$} & \multicolumn{2}{|c|}{$\mathrm{T} 2 \mathrm{D}(n=37)$} & \multirow{2}{*}{$p$ value } \\
\hline & & Mean or median & Increased no. (\%) & Mean or median & Increased no. (\%) & \\
\hline IL-2 & $\leq 11.4$ & $4.09 \pm 2.02$ & $0(0.00 \%)$ & $3.84 \pm 1.24$ & $0(0.00 \%)$ & 0.516 \\
\hline IL-4 & $\leq 12.9$ & $3.64 \pm 0.82$ & $0(0.00 \%)$ & $3.67 \pm 0.95$ & $0(0.00 \%)$ & 0.888 \\
\hline IL-6 & $\leq 20$ & 5.27 & $4(12.1 \%)$ & 12.10 & $9(24.3 \%)$ & $0.008^{*}$ \\
\hline IL-10 & $\leq 5.9$ & 4.50 & $8(24.2 \%)$ & 5.05 & $5.05(29.7 \%)$ & 0.187 \\
\hline TNF- $\alpha$ & $\leq 5.5$ & 3.83 & $6(18.2 \%)$ & 6.43 & $19(51.4 \%)$ & $<0.001^{*}$ \\
\hline IFN- $\gamma$ & $\leq 18$ & 3.59 & $2(6.1 \%)$ & 8.83 & $3(8.2 \%)$ & $0.005^{*}$ \\
\hline
\end{tabular}

in the pathological process of COVID-19 [5, 22-24]. The effectual host immune response including innate and adaptive immunity against SARS-CoV-2 is crucial to control and resolve the viral infection $[3,22,24]$. However, the severity and outcome of COVID-19 might also be associated with dysregulated immune response and excessive production of proinflammatory cytokines $[5,23,25,26]$. The immune system is impaired during the disease, characterized by leukocytopenia (esp. lymphocytopenia) and uncontrolled systemic inflammatory response in the severe cases [20, 23, 25]. Most recent data demonstrated that COVID-19 might affect lymphocytes, especially $\mathrm{T}$ lymphocytes [20, 21]. The absolute number of $\mathrm{T}$ lymphocytes, CD4+ T cells, and CD8+ T cells decreased in nearly all the patients and was markedly lower in severe cases $[5,20,27]$. And these potential immunological markers may be of importance due to their correlation with disease severity in COVID-19 [5, 23, 25, 26, 28].

T2D is well established as an inflammatory disease characterized by immune disturbance (e.g., long-term activation of the innate immune system) and systemic low-grade inflammation $[29,30]$. The basal immune status in T2D is dysregulated, which is believed to affect normal immune response against viral infection. In this retrospective pilot study, we made efforts to find out whether comorbidity with T2D was related to different immunological parameters during diagnosis and management of COVID-19. Patients with COVID-19 often had leukocytopenia, lymphocytopenia, and an increased monocyte count as previously reported $[1,2,20]$. However, we first revealed an abnormal increase in leukocyte (esp. neutrophil) count in patients comorbid with T2D compared with NDM. The T2D group also showed a significantly higher incidence of abnormal neutrophilia, which was primarily proven to be independent of secondary or combined bacterial infection. These data implied a dysregulated neutrophil response to SARS-CoV-2 infection in T2D patients. Neutrophil count was further established as an independent risk factor associated with severe (and critically ill) types of COVID-19, adjusted by age, sex, and mixed bacterial infection. Increased neutrophil or neutrophil-tolymphocyte ratio (NLR) is established as an independent risk factor and prognostic tool to predict the clinical outcomes of COVID-19 [22, 31-33]. Our data revealed that COVID-19 patients comorbid with T2D have distinguishable changes in the white cell subpopulation characterized by an intrinsically increased neutrophil count. And the abnormal increase in neutrophil count and its contribution to COVID-19 severity may be associated with innate dysregulation of immune response in T2D [33].

Previous studies had revealed that the dysregulation of cellular immune response, especially $\mathrm{T}$ lymphocytes, might be highly involved in the pathological process of COVID$19[20,25,27]$. Although the total lymphocyte count or the incidence of lymphopenia demonstrates no significant difference between the two groups, we observed distinguishable differences in the subpopulations of lymphocytes. The sustained decrease in total T cells as well as Th and Tc subsets and NK subsets were all more remarkable in the T2D group than in the nondiabetic group. Previous studies have demonstrated that the absolute number of $\mathrm{T}$ cell subsets was decreased in most patients, especially in severe cases [5, 20$22,25,27]$. Similar but not perfectly consistent tendencies were observed in the incidences of parameter abnormity in this study. $36.4 \%$ of patients without diabetes and $66.7 \%$ of T2D patients had a decreased Tc cell count. Patients from the T2D group also had a significantly higher incidence of decreased Tc cell proportion and NK cell count compared 
to those from the NDM group. And we found a significantly higher proportion but not a higher number of $\mathrm{B}$ cells in T2D patients. Conflictingly, T2D patients had a lower median level of Th cell count, but less patients in the T2D group had decreased Th cell proportion compared to those in the NDM group. This fact was due to a discrepant distribution of Th levels in T2D patients. And it reminded us to pay attention not only to the absolute levels of the immunological parameters but also to the incidence of abnormity in those parameters. Therefore, we analyzed differences in not only absolute values but also in the abnormal rates of the laboratory parameters between two subcohorts of patients. There were discrepant and even divergent cellular immune responses to SARS-CoV-2 infection in patients comorbid with T2D. And univariate analysis laid more stress on the relevance of the Th subset with disease severity in the current data. Th cell correlated positively with lymphocyte count, Tc count, B cell count, and NK cell count significantly, but correlated negatively with CRP levels and neutrophil count. Series of studies have confirmed that there is a skewed proinflammatory $\mathrm{T}$ cell compartment, especially Th subsets in the peripheral blood of T2D patients [34-36]. Th cells may play a double-edged role in antiviral response and uncontrolled inflammation. Besides, T2D is also accompanied by a baseline decrease in NK cell function [34,37]. And both cytotoxic lymphocytes including Tc and NK were more remarkably impaired in $\mathrm{T} 2 \mathrm{D}$ compared with nondiabetic patients in our study. Since Tc and NK interact intensively with the virus and play dominant roles in the clearance of virally infected cells, NK cells are also major immune regulators which bring order and discipline to the infected tissue microenvironment [38]. Decreased NK cell count may also explain the uncontrolled expansion and activation of other immune effectors $[20,38]$. Therefore, the dysregulated basal levels and discrepant response of lymphocyte subpopulations in T2D are postulated to have a critical role in the severity and adverse outcome of the disease $[20,39,40]$.

As an important regulatory immune component, Th cells regulate antigen presentation and immunity against intracellular SARS-CoV-2 through IFN- $\gamma$ production. Dysregulated $\mathrm{T}$ cell function relieves the inhibition on the innate immune system leading to secretion of high amounts of inflammatory cytokines in what is known as a "cytokine storm" [41]. We also investigated the Th1/Th2 cytokines in patients with and without diabetes. We did not observe statistical difference in the levels of the Th2 cytokines including IL-2, IL-4, and IL-10. However, serum levels of IFN- $\gamma$, TNF- $\alpha$, and IL6 , as well as CRP, were all significantly higher in the T2D group than in the NDM group. And increased CRP also correlated significantly with increased neutrophil count and negatively with lymphocyte, T cell, Th, Tc, B cell, and NK cell counts in this study. Therefore, Th1 cells may induce the recruitment of neutrophils and macrophages by producing inflammatory cytokines [42], which in turn contribute to the hyperinflammatory response observed in patients with diabetes [39]. Monoclonal antibody therapy targeting Th1 cytokines might be helpful especially in patients with T2D.

Moreover, T2D patients also demonstrate distinguishable differences in humoral immunity. COVID-19 patients had more often increased levels of $\operatorname{IgG}, \operatorname{IgE}$, and complement $\mathrm{C} 4$, but a decreased level of $\mathrm{C} 3$ in our study. The average levels of IgG, IgM, IgA, C3, and C4 showed no significant difference between the two groups. But abnormally higher $\operatorname{IgE}$ levels were observed in the T2D group. Serum IgE negatively correlated with Th and B cell counts significantly, but it positively correlated with neutrophil count in our cohort. Recent advances suggested that an increase in type 2 (antihelminths) effectors including IgE and eosinophils also reflected the development of a maladapted immune response profile and was associated with severe COVID-19 outcome [43]. These changes may represent a dysregulated humoral immune response in T2D and might be associated with the proinflammatory status and adverse outcome during infection [43].

In conclusion, the COVID-19 patients comorbid with T2D demonstrated distinguishable immunological parameters during the infection. Some of the discrepancies demonstrated clinical relevance with the predisposed adverse outcome of COVID-19 in T2D. Dysregulated baseline status and discrepant response of both cellular and humoral immunity in T2D are postulated to have a critical role in the severity and adverse outcome of the disease. The current data could shed some light on the understanding of the unique response to SARS-CoV-2 infection in T2D and thus provide useful clues for the development of more effective strategies.

\section{Data Availability}

The authors will make the primary data available under the premise of privacy protection, restricted usage, and duplication. Access to additional primary data will be considered by the authors upon request (rusingstar@163.com).

\section{Conflicts of Interest}

The authors declare that there are no conflicts of interest regarding the publication of this paper.

\section{Authors' Contributions}

Ruxing Zhao and Yujing Sun contributed equally to the work as first authors.

\section{Acknowledgments}

We acknowledge all the patients who participated in our study. And we would like to express our highest esteem and tremendous respect to those medical staff, organizations, and governments as well as general civilians who fought and who continue fighting against the global pandemic. This work was supported by the National Natural Science Foundation of China (Grant No. 81500631 to R.X.Z., Grant No. 81670706 to L.C., Grant No. 81770818 to X.G.H, and Grant No. 81873632 to L.C.), the Natural Science Foundation of Shandong Province (BS2015YY011 to R.X.Z.), the National Key R\&D Program of China (Grant No. 2018YFC1311801 to L.C.), and the COVID-19 emergency tackling research project of Shandong University (Grant No. 2020XGA 01 to Z.H.). 


\section{References}

[1] W. J. Guan, Z. Y. Ni, Y. Hu et al., "Clinical characteristics of coronavirus disease 2019 in China," The New England Journal of Medicine, vol. 382, no. 18, pp. 1708-1720, 2020.

[2] C. Huang, Y. Wang, X. Li et al., "Clinical features of patients infected with 2019 novel coronavirus in Wuhan, China," The Lancet, vol. 395, no. 10223, pp. 497-506, 2020.

[3] H. A. Rothan and S. N. Byrareddy, "The epidemiology and pathogenesis of coronavirus disease (COVID-19) outbreak," Journal of Autoimmunity, vol. 109, article 102433, 2020.

[4] X. Yang, Y. Yu, J. Xu et al., "Clinical course and outcomes of critically ill patients with SARS-CoV-2 pneumonia in Wuhan, China: a single-centered, retrospective, observational study," The Lancet Respiratory Medicine, vol. 8, no. 5, pp. 475-481, 2020.

[5] G. Chen, D. Wu, W. Guo et al., "Clinical and immunological features of severe and moderate coronavirus disease 2019," The Journal of Clinical Investigation, vol. 130, no. 5, pp. 2620-2629, 2020.

[6] Y. Bai, L. Yao, T. Wei et al., "Presumed asymptomatic carrier transmission of COVID-19," JAMA, vol. 323, no. 14, p. 1406, 2020.

[7] M. Lean, L. McCombie, and J. McSorely, "Trends in type 2 diabetes," BMJ, vol. 366, 2019.

[8] W. S. Azar, R. Njeim, A. H. Fares et al., "COVID-19 and diabetes mellitus: how one pandemic worsens the other," Reviews in Endocrine \& Metabolic Disorders, pp. 1-13, 2020.

[9] J. Pearson-Stuttard, S. Blundell, T. Harris, D. G. Cook, and J. Critchley, "Diabetes and infection: assessing the association with glycaemic control in population-based studies," The Lancet Diabetes and Endocrinology, vol. 4, no. 2, pp. 148-158, 2016.

[10] S. Ferlita, A. Yegiazaryan, N. Noori et al., "Type 2 diabetes mellitus and altered immune system leading to susceptibility to pathogens, especially Mycobacterium tuberculosis," Journal of Clinical Medicine, vol. 8, no. 12, p. 2219, 2019.

[11] Z. T. Bloomgarden, “Diabetes and COVID-19," Journal of Diabetes, vol. 12, no. 4, pp. 347-348, 2020.

[12] R. Gupta, A. Ghosh, A. K. Singh, and A. Misra, "Clinical considerations for patients with diabetes in times of COVID-19 epidemic," Diabetes and Metabolic Syndrome: Clinical Research and Reviews, vol. 14, no. 3, pp. 211-212, 2020.

[13] R. Pal and S. K. Bhadada, "COVID-19 and diabetes mellitus: an unholy interaction of two pandemics," Diabetes and Metabolic Syndrome: Clinical Research and Reviews, vol. 14, no. 4, pp. 513-517, 2020.

[14] A. M. Angelidi, M. J. Belanger, and C. S. Mantzoros, "Commentary: COVID-19 and diabetes mellitus: what we know, how our patients should be treated now, and what should happen next," Metabolism, vol. 107, article 154245, 2020.

[15] S. R. Bornstein, F. Rubino, K. Khunti et al., "Practical recommendations for the management of diabetes in patients with COVID-19," The Lancet Diabetes \& Endocrinology, vol. 8, no. 6, pp. 546-550, 2020.

[16] L. Fang, G. Karakiulakis, and M. Roth, "Are patients with hypertension and diabetes mellitus at increased risk for COVID-19 infection?," The Lancet Respiratory Medicine, vol. 8 , no. 4, article e21, 2020.

[17] R. Gupta, A. Hussain, and A. Misra, "Diabetes and COVID-19: evidence, current status and unanswered research questions,"
European Journal of Clinical Nutrition, vol. 74, no. 6, pp. 864-870, 2020.

[18] Q. Shi, X. Zhang, F. Jiang et al., "Clinical characteristics and risk factors for mortality of COVID-19 patients with diabetes in Wuhan, China: a two-center, retrospective study," Diabetes Care, vol. 43, no. 7, pp. 1382-1391, 2020.

[19] M. C. Riddle, J. B. Buse, P. W. Franks et al., "COVID-19 in people with diabetes: urgently needed lessons from early reports," Diabetes Care, vol. 43, no. 7, pp. 1378-1381, 2020.

[20] C. Qin, L. Zhou, Z. Hu et al., "Dysregulation of immune response in patients with coronavirus 2019 (COVID-19) in Wuhan, China," Clinical Infectious Diseases, vol. 71, no. 15, pp. 762-768, 2020.

[21] J. Liu, S. Li, J. Liu et al., "Longitudinal characteristics of lymphocyte responses and cytokine profiles in the peripheral blood of SARS-CoV-2 infected patients," eBioMedicine, vol. 55, p. 102763, 2020.

[22] R. Chen, L. Sang, M. Jiang et al., "Longitudinal hematologic and immunologic variations associated with the progression of COVID-19 patients in China," The Journal of Allergy and Clinical Immunology, vol. 146, no. 1, pp. 89-100, 2020.

[23] X. Li, S. Xu, M. Yu et al., "Risk factors for severity and mortality in adult COVID-19 inpatients in Wuhan," The Journal of Allergy and Clinical Immunology, vol. 146, no. 1, pp. 110$118,2020$.

[24] E. Maggi, G. W. Canonica, and L. Moretta, "COVID-19: unanswered questions on immune response and pathogenesis," The Journal of Allergy and Clinical Immunology, vol. 146, no. 1, pp. 18-22, 2020.

[25] R. He, Z. Lu, L. Zhang et al., "The clinical course and its correlated immune status in COVID-19 pneumonia," Journal of Clinical Virology, vol. 127, p. 104361, 2020.

[26] T. Herold, V. Jurinovic, C. Arnreich et al., "Elevated levels of IL-6 and CRP predict the need for mechanical ventilation in COVID-19," The Journal of Allergy and Clinical Immunology, vol. 146, no. 1, pp. 128-136.e4, 2020.

[27] F. Wang, J. Nie, H. Wang et al., "Characteristics of peripheral lymphocyte subset alteration in COVID-19 pneumonia," The Journal of Infectious Diseases, vol. 221, no. 11, pp. 17621769, 2020.

[28] Y. Yang, C. Shen, J. Li et al., "Plasma IP-10 and MCP-3 levels are highly associated with disease severity and predict the progression of COVID-19," The Journal of Allergy and Clinical Immunology, vol. 146, no. 1, pp. 119-127.e4, 2020.

[29] M. Prasad, E. W. Chen, S. A. Toh, and N. R. J. Gascoigne, "Autoimmune responses and inflammation in type 2 diabetes," Journal of Leukocyte Biology, vol. 107, no. 5, pp. 739$748,2020$.

[30] M. Y. Donath, C. A. Dinarello, and T. Mandrup-Poulsen, "Targeting innate immune mediators in type 1 and type 2 diabetes," Nature Reviews Immunology, vol. 19, no. 12, pp. 734746, 2019.

[31] J. Lian, C. jin, S. Hao et al., "High neutrophil-to-lymphocyte ratio associated with progression to critical illness in older patients with COVID-19: a multicenter retrospective study," Aging, vol. 12, no. 14, pp. 13849-13859, 2020.

[32] Y. Liu, X. du, J. Chen et al., "Neutrophil-to-lymphocyte ratio as an independent risk factor for mortality in hospitalized patients with COVID-19," The Journal of Infection, vol. 81, no. 1, pp. e6-e12, 2020. 
[33] C. Wu, et al.X. Chen, Y. Cai et al., "Risk factors associated with acute respiratory distress syndrome and death in patients with coronavirus disease 2019 pneumonia in Wuhan, China," JAMA Internal Medicine, vol. 180, no. 7, p. 934, 2020.

[34] M. Jagannathan-Bogdan, M. E. McDonnell, H. Shin et al., "Elevated proinflammatory cytokine production by a skewed T cell compartment requires monocytes and promotes inflammation in type 2 diabetes," Journal of Immunology, vol. 186, no. 2, pp. 1162-1172, 2011.

[35] C. Zeng, X. Shi, B. Zhang et al., "The imbalance of Th17/Th1/Tregs in patients with type 2 diabetes: relationship with metabolic factors and complications," Journal of Molecular Medicine, vol. 90, no. 2, pp. 175-186, 2012.

[36] R. Zhao, D. Tang, S. Yi et al., "Elevated peripheral frequencies of Th22 cells: a novel potent participant in obesity and type 2 diabetes," PLoS One, vol. 9, no. 1, article e85770, 2014.

[37] J. H. Kim, K. Park, S. B. Lee et al., "Relationship between natural killer cell activity and glucose control in patients with type 2 diabetes and prediabetes," Journal of Diabetes Investigation, vol. 10, no. 5, pp. 1223-1228, 2019.

[38] A. Jewett, "The potential effect of novel coronavirus SARSCoV-2 on NK cells; a perspective on potential therapeutic interventions," Frontiers in Immunology, vol. 11, p. 1692, 2020.

[39] R. Muniyappa and S. Gubbi, "COVID-19 pandemic, coronaviruses, and diabetes mellitus," American Journal of Physiology-Endocrinology and Metabolism, vol. 318, no. 5, pp. E736-E741, 2020.

[40] K. S. Rao, V. Suryaprakash, R. Senthilkumar et al., "Role of immune dysregulation in increased mortality among a specific subset of COVID-19 patients and immune-enhancement strategies for combatting through nutritional supplements," Frontiers in Immunology, vol. 11, p. 1548, 2020.

[41] N. W. Palm and R. Medzhitov, "Not so fast: adaptive suppression of innate immunity," Nature Medicine, vol. 13, no. 10, pp. 1142-1144, 2007.

[42] E. de Wit, N. van Doremalen, D. Falzarano, and V. J. Munster, "SARS and MERS: recent insights into emerging coronaviruses," Nature Reviews Microbiology, vol. 14, no. 8, pp. 523-534, 2016.

[43] C. Lucas, P. Wong, J. Klein et al., "Longitudinal analyses reveal immunological misfiring in severe COVID-19," Nature, vol. 584, no. 7821, pp. 463-469, 2020. 